\section{(2) OPEN ACCESS}

\title{
Income level and outcomes in patients with heart failure with universal health coverage
}

\author{
Chung-Lieh Hung (1) , 1,2,3 Tze-Fan Chao, ${ }^{4,5}$ Cheng-Huang Su, ${ }^{1,2,3}$ Jo-Nan Liao, ${ }^{4,5}$ \\ Kuo-Tzu Sung, ${ }^{1,2,3}$ Hung-I Yeh, ${ }^{1,2,3}$ Chern-En Chiang ${ }^{4,5,6,7}$
}

- Additional material is published online only. To view please visit the journal online (http://dx.doi.org/10.1136/ heartjnl-2020-316793).

For numbered affiliations see end of article.

\section{Correspondence to} Professor Chern-En Chiang, General Clinical Research Center, Taipei Veterans General Hospital, Taipei 112, Taiwan: cechiang@vghtpe.gov.tw

C-LH and T-FC contributed equally.

C-LH and T-FC are joint first authors.

Received 26 February 2020 Revised 3 September 2020 Accepted 10 September 2020 Published Online First 20 October 2020

\section{Linked}

- http://dx.doi.org/10.1136/ heartjnl-2020-317977

Check for updates

(C) Author(s) (or their employer(s)) 2021. Re-use permitted under CC BY-NC. No commercial re-use. See rights and permissions. Published by BMJ.

To cite: Hung C-L, Chao TF, Su C-H, et al. Heart 2021;107:208-216.

\section{ABSTRACT \\ Objective We aimed to investigate the influence of income level on guideline-directed medical therapy (GDMT) prescription rates and prognosis of patients with heart failure (HF) following implementation of a nationwide health insurance programme.}

Methods A total of 633098 hospitalised patients with HF from 1996 to 2013 were identified from Taiwan National Health Insurance Research Database. Participants were classified into low-income, medianincome and high-income groups. GDMT utilisation, in-hospital mortality and postdischarge HF readmission, and mortality rates were compared.

Results The low-income group had a higher comorbidity burden and was less likely to receive GDMT than the other two groups. The in-hospital mortality rate in the low-income group (5.07\%) was higher than in the median-income $(2.47 \%)$ and high-income $(2.51 \%)$ groups. Compared with the high-income group, the low-income group had a significantly higher risk of postdischarge HF readmission (adjusted HR (aHR): 1.29, $95 \% \mathrm{Cl} 1.27$ to 1.31), all-cause mortality (aHR: 1.98 , $95 \% \mathrm{Cl} 1.95$ to 2.02 ) and composite $\mathrm{HF}$ readmission/allcause mortality (aHR: 1.54, 95\% Cl 1.52 to 1.56). These results were generally consistent among the population after propensity matching (low vs high: HR=2.08 for mortality and 1.36 for HF readmission; median vs high: $H R=1.23$ for mortality and 1.12 for $\mathrm{HF}$ readmission; all $p<0.001$ ) and after inverse probability of treatment weighting (low-income vs high-income group: HR: 2.19 for mortality and 1.16 for HF readmission; medianincome vs high-income group: HR: 1.53 for mortality and 1.09 for $\mathrm{HF}$ readmission; all $p<0.001)$. Lower utilisation of GDMT and poorer prognosis in lower-income hospitalised patients with $\mathrm{HF}$ appeared to mitigate over time.

Conclusions Low-income patients with HF had nearly a twofold increase in the risk of in-hospital mortality and postdischarge events compared with the highincome group, partly due to lower GDMT utilisation. The differences between postdischarge HF outcomes among various income groups appeared to mitigate over time following the implementation of nationwide universal health coverage.

\section{INTRODUCTION}

Heart failure (HF) emerges as a global threat in all cardiovascular diseases, ${ }^{1}$ especially in hospitalised patients, leading to high morbidity and mortality. ${ }^{2}$ HF inflicts a considerable economic burden on the healthcare system worldwide, not merely in
Western nations but also in the Asia-Pacific regions, particularly in low-income and middle-income countries. ${ }^{3}$ As the final pathway of most cardiovascular disorders ${ }^{4}$ and the leading cause of hospitalisation among adults and the elderly population, the prevalence and burden of HF will continue to rise (up to 25\%) in the next two decades in both developing and developed countries. ${ }^{1}$

It is generally believed that individuals with lower socioeconomic status are much more likely to develop heart disease than those who are wealthier. ${ }^{5}$ The poorer prognosis of patients with HF with lower income may be due to the misallocation of medical resources and differences in education level, degree of urbanisation, ability for self-care, wealth, environment and family support. Shorter life expectancy in HF was observed regardless of gender or ethnicity in developing countries, such as some Asian countries, ${ }^{6}$ partly attributable to highly diverse quality and performance of healthcare (ie, evidence-based therapies) across different socioeconomic regions. A healthcare system with universal coverage of health (UCH) insurance, for example, implementation of nationwide healthcare system, would be expected to eliminate gaps and variations of healthcare quality among subjects with different income levels and sociodemographic backgrounds within the same society, and therefore might theoretically improve clinical endpoints. ${ }^{7}$

Taiwan as a unique country paved the way for $\mathrm{UCH}$ by establishing a unique healthcare system for universal health coverage (National Health Insurance (NHI)) for more than two decades by assuring equal access to healthcare resources for all citizens (ie, guideline-directed medical therapy (GDMT)) regardless of socioeconomic level. ${ }^{8}$ By reviewing data regarding the temporal transitions of several key outcome measures, we may find evidence reflecting efficacy following enforcement of the nationwide healthcare insurance from the country level. In the present study, we aimed to investigate the impact of income level on the prognosis of patients with HF at the nationwide level.

\section{METHODS \\ Database}

This study used data from the National Health Insurance Research Database (NHIRD), released by the Taiwan National Health Research Institutes. The NHI system is a universal, governmentendorsed health insurance programme passed in 1994 and launched in 1995 that offers 
comprehensive medical care coverage to nearly all (>99.99\%) Taiwanese population, with the NHI Administration overseeing the plan and controlling the global expenditure. ${ }^{9}$ The NHIRD collects detailed healthcare data from more than 23 million NHI enrollees in Taiwan. More information regarding the NHI system in Taiwan and categorisation of patients' income level (as low: <20000; median: 20000-39 999; and high: $\geq 40000$ new Taiwan dollars) are further detailed in online supplemental materials. In this cohort data set, patients' original identification numbers were encrypted to protect their privacy; however, the encrypting procedure was consistent so the claims belonging to the same patient could be linked within the NHI database and patients could be followed up. ${ }^{810}$

\section{Study population}

From 1 January 1996 to 31 December 2013, a total of 633098 subjects aged 20 or older with a diagnosis of HF hospitalisation, according to the International Classification of Diseases, Ninth Revision, Clinical Modification (ICD-9-CM) codes 428.0-428.4, 428.9 , without coexistence of a main diagnosis of acute coronary syndrome, were identified from the NHIRD. Information on important comorbid conditions for each individual was also retrieved from the NHIRD based on the ICD-9-CM codes. The diagnostic accuracy of important comorbidities in the NHIRD, including hypertension, diabetes mellitus, myocardial infarction, hyperlipidaemia and chronic obstructive pulmonary disease, has been previously validated, ${ }^{1011}$ with Charlson Comorbidity Index (CCI) used to represent the comorbidity burden of the patients. ${ }^{12}$

\section{Clinical outcomes}

The clinical outcomes of the present study included in-hospital mortality and postdischarge HF readmission and composite outcome of all-cause mortality and HF readmission assessed following the index date of adjudicated HF discharge in survivors. Validity of the main outcome measures in the current study is detailed in the online supplemental materials. The temporal trends of events (HF readmission or all-cause mortality) were investigated. The risk of events was compared between the different income groups.

\section{Propensity matching analysis}

We performed propensity score-matched analyses for two kinds of comparisons: low-income versus high-income, and medianincome versus high-income, conditional on all key baseline covariates listed in table 1. Online supplemental figures 1 and 2 show the distributions of propensity scores of study subjects for being as low-income and median-income groups before and after the propensity match, respectively. To show consistency of the estimates after matching, alternative matching methods were conducted using inverse probability of treatment weighting (IPTW). Methods on these matching processes are detailed in online supplemental materials.

\section{Statistical analysis}

Data were summarised using mean and SD for continuous variables and proportions for categorical variables. Group differences for continuous values were assessed using unpaired two-tailed t-tests or one-way analysis of variance. Group differences for nominal variables were compared using $\chi^{2}$. An interaction analysis was performed by adding an interaction term to a regression model between income strata and three major time intervals (1996-2001, 2002-2007 and 2008-2013) as a continuous linear predictor with respect to CCI (age-adjusted and sex-adjusted). A linear regression analysis was used to test the linear trends of CCI (age-adjusted and sex-adjusted), HF pharmacological prescription patterns and in-hospital mortality (expressed as adjusted ORs for median-income/high-income groups, and low-income as reference) across three major time intervals as ordinal category. The survival function estimating the risk of HF readmission, all-cause mortality and composite outcome of HF readmission/mortality postdischarge was assessed using Cox regression analysis. The risk of in-hospital mortality was assessed using logistic regression analysis. The cumulative incidence curve of all-cause mortality was plotted using the Kaplan-Meier method, with statistical significance examined with the log-rank test. Subgroup analyses for HF outcomes using Cox regression models among income strata (median-income/ low-income vs high-income group) were conducted according to key baseline characteristics (including age, gender, degree of urbanisation, comorbidities and HF-related medications). Statistical significance was set at $\mathrm{p}<0.05$. All analyses were performed using IBM SPSS Statistics for Windows V.20.0 and SAS software V.9.4.

\section{Patient and public involvement}

Participants were not involved in the design, conduct, reporting or dissemination plans of our research.

\section{RESULTS}

\section{Baseline demographics}

Baseline characteristics are displayed in table 1. Among 633098 patients hospitalised with HF from 1996 to 2013, 401639 (63.4\%) were categorised as low income, $190167(30.0 \%)$ as median income and $41292(6.5 \%)$ as high income. The mean age of $\mathrm{HF}$ diagnosis was $71.7(\mathrm{SD}=13.4)$ years, and gender was nearly equally distributed $(51.1 \% \mathrm{men})$. There was a significant difference $(\mathrm{p}<0.001)$ in mean age between the income groups: 58.9 (12.6) years in the high-income, 68.3 (14.5) in the medianincome and 74.6 (11.7) in the low-income group. In our study cohort, patients with HF with low income were older, more likely to be female, more likely to have a history of stroke/ transient ischaemic attack and chronic obstructive pulmonary disease, less likely to have vascular diseases (including coronary artery disease), chronic kidney disease and hyperlipidaemia, and more likely to live in rural regions, compared with medianincome and high-income groups. CCI was higher in low-income and median-income groups than in high-income patients with HF (6.4 and 6.78 vs $6.11, \mathrm{p}<0.001$; table 1 ).

\section{Association between income level, comorbidity burden and pharmacological use}

Comorbidity burden as measured by CCI increased in a graded fashion from 1996 to 2013 (classified into 1996-2001, 20022007 and 2008-2013) for all patients with HF postdischarge irrespective of income strata (all $\mathrm{p}_{\text {trend }}<0.001$; figure 1 ). The age-adjusted and sex-adjusted CCI increment over time was 1.01 (95\% CI 0.99 to 1.02 per decade, $p<0.001)$ and was most pronounced in the low-income group, followed by the medianincome and high-income groups (1.49 (95\% CI 1.47 to 1.51), 0.2 (95\% CI 0.17 to 0.23 ), 0.36 (95\% CI 0.29 to 0.42 ) per decade for low-income, median-income and high-income HF groups, respectively; $\mathrm{p}_{\text {interaction }}<0.001$ ), indicating a temporal trend of increasing comorbidity burden in discharged patients with HF over time particularly in the low-income group.

We also observed different prescription patterns for several HF-related medications across the income groups. Low-income 


\begin{tabular}{|c|c|c|c|c|c|}
\hline Income groups & All & Low-income & Median-income & High-income & $P$ value \\
\hline$n$ & 633098 & 401639 & 190167 & 41292 & \\
\hline Age, years, mean (SD) & 71.7 (13.4) & 74.6 (11.7) & $68.3(14.5)$ & 58.9 (12.6) & $<0.001$ \\
\hline$\geq 75, \mathrm{n}(\%)$ & $308705(48.8)$ & 231539 (57.6) & $72761(38.3)$ & 4405 (10.7) & $<0.001$ \\
\hline $65-74$, n (\%) & 165987 (26.2) & 107431 (26.7) & $49760(26.2)$ & $8796(21.3)$ & $<0.001$ \\
\hline Charlson Comorbidity Index, mean (SD) & $6.49(2.98)$ & $6.40(2.97)$ & $6.78(2.98)$ & $6.11(3.06)$ & $<0.001$ \\
\hline \multicolumn{6}{|l|}{ Comorbidities, n (\%) } \\
\hline Hypertension & 482638 (76.2) & $300199(74.7)$ & $151610(79.7)$ & $30829(74.7)$ & $<0.001$ \\
\hline Diabetes mellitus & $258863(40.9)$ & $160734(40.0)$ & $80407(42.3)$ & $17722(42.9)$ & $<0.001$ \\
\hline Stroke/TIA & $181724(28.7)$ & $119711(29.8)$ & $53108(27.9)$ & $8905(21.6)$ & $<0.001$ \\
\hline Vascular diseases & $368897(58.3)$ & $226478(56.4)$ & $117955(62.0)$ & $24464(59.2)$ & $<0.001$ \\
\hline Autoimmune diseases & $41480(6.6)$ & $23854(5.9)$ & $15108(7.9)$ & $2518(6.1)$ & $<0.001$ \\
\hline Liver cirrhosis & $29717(4.7)$ & $18246(4.5)$ & $9576(5.0)$ & $1895(4.6)$ & $<0.001$ \\
\hline Dyslipidaemia & $195356(30.9)$ & $103933(25.9)$ & $73023(38.4)$ & $18400(44.6)$ & $<0.001$ \\
\hline CKD & $125624(19.8)$ & $75543(18.8)$ & $40527(21.3)$ & $9554(23.1)$ & $<0.001$ \\
\hline VHD & $40031(6.3)$ & $23816(5.9)$ & $13499(7.1)$ & $2716(6.6)$ & $<0.001$ \\
\hline Anaemia & $158116(25.0)$ & $101442(25.3)$ & $48959(25.7)$ & $7715(18.7)$ & 0.001 \\
\hline Valvular heart surgery & $4053(0.6)$ & $1565(0.4)$ & $1717(0.9)$ & $771(1.9)$ & $<0.001$ \\
\hline CABG & $12349(2.0)$ & $6337(1.6)$ & $4202(2.2)$ & $1810(4.4)$ & $<0.001$ \\
\hline AF & $118744(18.8)$ & $74111(18.5)$ & $37075(19.5)$ & $7558(18.3)$ & $<0.001$ \\
\hline Degree of urbanisation, $n(\%)$ & & & & & $<0.001$ \\
\hline Urban & $309424(48.9)$ & $209448(52.1)$ & $71285(37.5)$ & 28691 (69.5) & \\
\hline Suburban & $203913(32.2)$ & $127761(31.8)$ & $64879(34.1)$ & $11273(27.3)$ & \\
\hline Diuretics & $336887(53.2)$ & $219954(54.8)$ & $97190(51.1)$ & $19743(47.8)$ & $<0.001$ \\
\hline $\mathrm{MRA}^{*}$ & $106170(16.8)$ & $61396(15.3)$ & $36407(19.1)$ & $8367(20.3)$ & $<0.001$ \\
\hline
\end{tabular}

*MRA excluded.

ACEIs, ACE inhibitors; : AF, atrial fibrillation; ARBs, angiotensin receptor blockers; CABG, coronary artery bypass graft; CKD, chronic kidney disease; COPD, chronic obstructive pulmonary disease; ESRD, end-stage renal disease; MRA, mineralocorticoid receptor antagonist (eplerenone/spironolactone); TIA, transient ischaemic attack; VHD, valvular heart disease.

patients with HF were less frequently prescribed GDMT for reduced ejection fraction HF (ie, ACE inhibitors/angiotensin receptor blockers (ACEIs/ARBs), beta-blockers (BBs) and mineralocorticoid receptor antagonists (MRAs)) and amiodarone, although they were more likely to receive digoxin and diuretics when compared with middle-income and high-income $\mathrm{HF}$ groups (all $\mathrm{p}<0.001$; figure 2). These findings indicate a different pharmacological prescription pattern of HF medications across different income strata. Overall, the differences in HF pharmacological prescription patterns among income groups decreased in fully adjusted models (as adjusted ORs, with low-income as reference) across time intervals (1996-2001, 2002-2007 and 2008-2013) (all $\left.\mathrm{p}_{\text {trend }}<0.001\right)$ (online supplemental table 1).

\section{Association between income level and in-hospital mortality}

Among 633098 patients aged 20 or older between 1996 and 2013 with HF hospitalisation, 26093 (4.1\%) died during admission. A significantly higher in-hospital mortality rate was observed in the low-income $(5.07 \%)$ compared with the median-income $(2.47 \%)$ and high-income $(2.51 \%)$ HF groups (table 1). The risk of in-hospital mortality was significantly higher for the low-income HF population (crude OR: 2.07 (95\% CI 1.94 to 2.21), $\mathrm{p}<0.05$; table 2 ) and remained significant in the fully adjusted model (adjusted OR: 1.53 (95\% CI 1.43 to 1.64), $\mathrm{p}<0.05$; table 2). Differences in in-hospital mortality for median-income and high-income groups compared with low-income group also decreased (as adjusted ORs) across time intervals (1996-2001, 2002-2007 and 2008-2013) (both $\mathrm{p}_{\text {trend }}$ $<0.001$ ) in fully adjusted models (figure 3).

\section{Association between income level and HF outcomes}

Among the total 607005 discharged HF survivors, all-cause mortality, HF readmission, and composite all-cause mortality and HF readmission were observed in 391337 (64.5\%), 287226 (47.3\%), and $476425(78.5 \%)$ patients, respectively, during the study observation period. The cumulative incidence curves of postdischarge $\mathrm{HF}$ readmission and $\mathrm{HF}$ readmission/mortality are shown in figure $4 \mathrm{~A}$ and $\mathrm{B}$, respectively. Overall, 16.8\%, 15.6\% and $17.4 \%$ of mortality/HF readmission cases occurred within the first month (30 days) postdischarge across the three income 


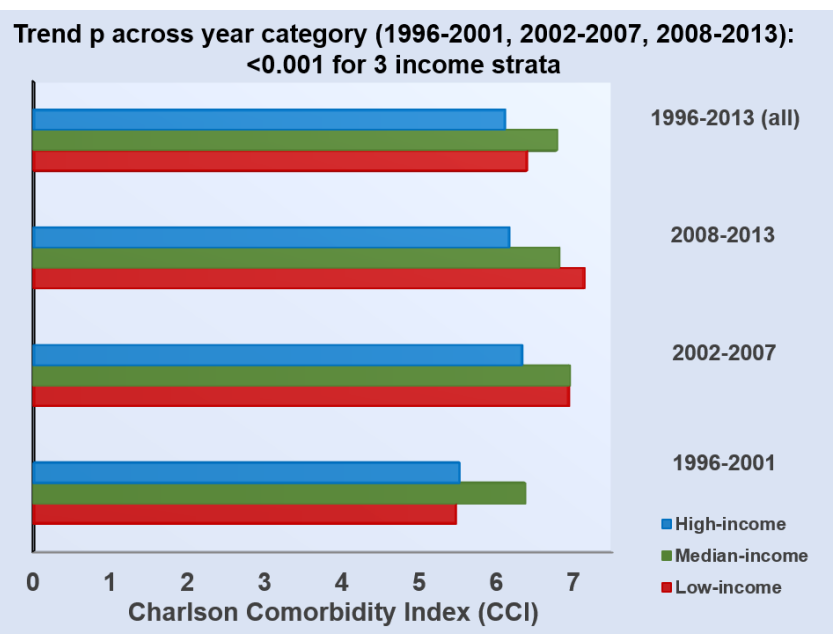

Figure 1 Charlson Comorbidity Index (CCI) stratified by three income groups. $\mathrm{CCl}$ increased in a graded fashion for all postdischarge patients with HF over time (classified into 1996-2001, 2002-2007 and 20082013) irrespective of income strata (all $p_{\text {trend }}<0.001$ ).

groups (for low-income, median-income and high-income HF groups, respectively). Similar trends in $\mathrm{HF}$ readmission or mortality were also observed (table 2). Notably, the temporal trends of risk of HF readmission or mortality in the low-income group diminished markedly after nearly one decade from the initiation of the NHI programme (HR of HF readmission and composite HF readmission/mortality: 2.64 and 4.94 in 1996 vs 1.46 and 2.65 in 2008 for the low-income group, using highincome group as reference; $\mathrm{p}_{\text {trend }}<0.001$; figure $5 \mathrm{~A}$ and B). Findings from subgroup analyses are shown in online supplemental figure 3 , with details provided in online supplemental materials.

\section{Propensity analysis}

The baseline characteristics of patients after matching are shown in online supplemental table 2 . The propensity scores did not

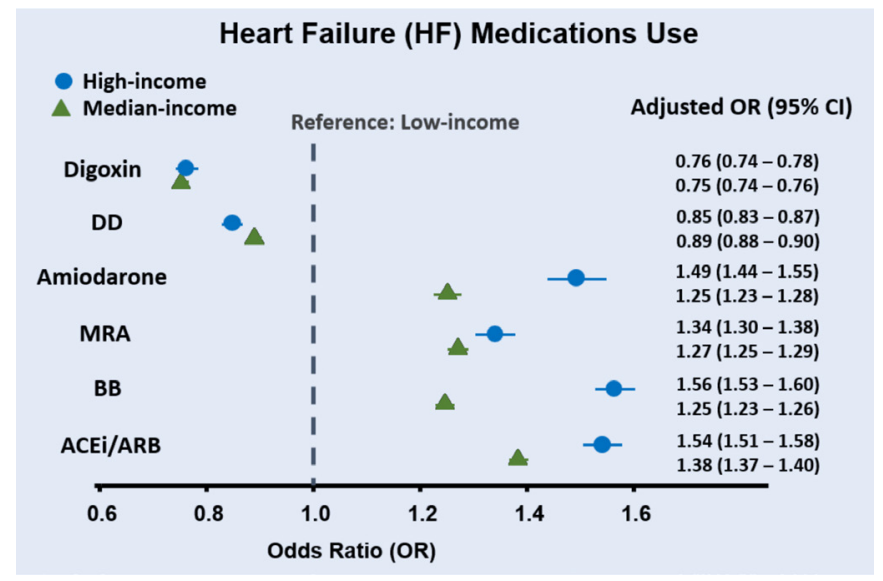

Figure 2 Heart failure medications stratified by three income groups. Different patterns of medication use across income groups were observed, with the median-income and high-income groups being more likely to receive GDMT (including ACEi/ARB, BB and MRA) and amiodarone, and less commonly prescribed DD (MRA excluded) and digoxin in fully adjusted models. ACEi, ACE inhibitor; ARB, angiotensin receptor blocker; $\mathrm{BB}$, beta-blocker; $\mathrm{DD}$, diuretic drugs; $\mathrm{GDMT}$, guidelinedirected medical therapy; MRA, mineralocorticoid receptor antagonist (eplerenone/spironolactone). differ significantly for low-income versus high-income group, and median-income versus high-income group. Comparisons of in-hospital mortality after propensity matching are shown in online supplemental table 2. Postdischarge HF readmission and mortality remained the lowest in the high-income group compared with the median-income group (median-income vs high-income: $\mathrm{HR}=1.23$ (1.20-1.25) for mortality, HR: 1.12 (1.10-1.15) for HF readmission) and low-income group (lowincome vs high-income: HR: 2.08 (2.04-2.13) for mortality, HR: $1.36(1.33-1.39)$ for HF readmission; all $\mathrm{p}<0.001)$ after matching (online supplemental table 3 ). The results of various subgroup analyses of outcomes by different income strata were broadly consistent after matching (online supplemental figure 3). Temporal changes on main outcome measures show similar trends as shown in online supplemental figure 4. Subgroup analyses were broadly similar after matching (online supplemental figure 3).

Baseline characteristics of patients after IPTW are shown in online supplemental table 4. After weighting, the three groups were well balanced in most characteristics (absolute standardised mean difference $<0.1$ ). The main outcome measures after IPTW remained the lowest in the high-income group compared with the median-income group (median-income vs high-income: HR: 1.53 (1.26-1.75) for mortality, 1.09 (1.05-1.25) for HF readmission) and low-income group (low-income vs high-income: HR: 2.19 (2.07-2.86) for mortality, 1.16 (1.08-1.35) for HF readmission; all $\mathrm{p}<0.001$ ) (online supplemental table 5).

\section{DISCUSSION}

In a nationwide data set with nearly full coverage of healthcare insurance, we investigated the temporal trends of comorbidity burden, GDMT utilisation and prognosis among discharged patients with HF with various sociodemographic backgrounds. The main findings of our study are as follows: Patients with HF with lower income had a markedly higher comorbidity burden, less likely to receive GDMT and showed a twofold increased risk of in-hospital mortality, along with nearly threefold and 1.5 -fold increased risk of postdischarge HF readmission and allcause mortality, even after correction for several key baseline demographic information. These findings were broadly consistent after propensity matching. Second, there appeared to be a temporal trend of mitigated variations of GDMT utilisation and postdischarge HF prognosis across different income strata about one decade following implementation of the nationwide healthcare insurance, despite an overall increase in comorbidity burden among all patients with HF.

Prior reports consistently found that socioeconomically deprived individuals might show a higher incidence of HF. ${ }^{13}$ The causal relationship between lower socioeconomic status and poorer prognosis has also been confirmed from a longitudinal study exploring income changes with incident cardiovascular events including $\mathrm{HF}^{14} \mathrm{~A}$ recent global between-country analysis showed that income inequality, rather than income level alone, may impact on HF outcomes to a similar degree as do major comorbidities. ${ }^{15}$ The relationship between lower socioeconomic status and worse clinical outcome could be bidirectional due to higher economic burden imposed by HF per se or HF-related comorbidities. Our findings were consistent with prior reports in that poorer prognosis is more likely to occur in lower-income patients with HF, presumptively explained by multiple influences from sociodemographic diversity including healthcare access, quality of practice, barriers to evidence-based care and underlying nutritional status. ${ }^{16-18}$ Findings of markedly older 
Table 2 Incidence of all-cause mortality, HF readmission and composite endpoint in patients with heart failure

\begin{tabular}{|c|c|c|c|c|}
\hline Income groups & Total & High-income & Median-income & Low-income \\
\hline Patients, $\mathrm{n}$ & 633098 & 41292 & 190167 & 401639 \\
\hline In-hospital mortality, n & 26093 & 1038 & 4703 & 20352 \\
\hline Events rate, $\%$ & 4.12 & 2.51 & 2.47 & 5.07 \\
\hline Unadjusted OR $(95 \% \mathrm{Cl})$ & - & - & $0.98(0.92$ to 1.05$) \dagger$ & $2.07(1.94$ to 2.21$) \dagger$ \\
\hline Model 1: OR $(95 \% \mathrm{Cl})$ & - & - & $0.95(0.89$ to 1.02$) \dagger$ & $1.92(1.80$ to 2.05$) \dagger$ \\
\hline Model 2: OR $(95 \% \mathrm{Cl})$ & - & - & $0.96(0.90$ to 1.03$) \dagger$ & $1.53(1.43$ to 1.64$) \dagger$ \\
\hline All-cause mortality, $\mathrm{n}$ & 391337 & 12872 & 85797 & 292668 \\
\hline Person-years & 2454689 & 201095 & 898631 & 1354963 \\
\hline Incidence* & 15.94 (15.89 to 15.99$)$ & 6.40 (6.29 to 6.51$)$ & 9.55 (9.48 to 9.61$)$ & 21.60 (21.52 to 21.68$)$ \\
\hline Unadjusted HR (95\% Cl) & - & - & $1.48(1.46$ to 1.51$) \dagger$ & $3.16(3.10$ to 3.21$) \dagger$ \\
\hline Model 1: HR $(95 \% \mathrm{Cl})$ & - & - & $1.13(1.11$ to 1.15$) \dagger$ & $1.99(1.96$ to 2.03$) \dagger$ \\
\hline Model 2: HR (95\% Cl) & - & - & $1.16(1.14$ to 1.18$) \dagger$ & $1.98(1.95$ to 2.02$) \dagger$ \\
\hline HF readmission, $n$ & 287226 & 16255 & 85954 & 185017 \\
\hline Person-years & 1593620 & 140944 & 596458 & 856217 \\
\hline Incidence* & 18.02 (17.96 to 18.09$)$ & 11.53 (11.36 to 11.71$)$ & 14.41 (14.31 to 14.51$)$ & 21.61 (21.51 to 21.71$)$ \\
\hline Unadjusted HR $(95 \% \mathrm{Cl})$ & - & - & $1.20(1.18$ to 1.22$) \dagger$ & $1.55(1.53$ to 1.58$) \dagger$ \\
\hline Model 1: HR $(95 \% \mathrm{Cl})$ & - & - & $1.08(1.06$ to 1.10$) \dagger$ & $1.28(1.26$ to 1.30$) \dagger$ \\
\hline Model 2: HR (95\% Cl) & - & - & $1.08(1.06$ to 1.09$) \dagger$ & $1.29(1.27$ to 1.31$) \dagger$ \\
\hline All-cause mortality/HF readmission, $n$ & 476425 & 22425 & 124745 & 329255 \\
\hline Person-years & 1593618 & 140944 & 596458 & 856216 \\
\hline Incidence* & 29.90 (29.81 to 29.98$)$ & 15.91 (15.70 to 16.12$)$ & 20.91 (20.80 to 21.03$)$ & 38.45 (38.32 to 38.59$)$ \\
\hline Unadjusted HR $(95 \% \mathrm{Cl})$ & - & - & $1.27(1.25$ to 1.29$) \dagger$ & $2.04(2.02$ to 2.07$) \dagger$ \\
\hline Model 1: HR $(95 \% \mathrm{Cl})$ & - & - & $1.08(1.07$ to 1.10$) \dagger$ & $1.55(1.53$ to 1.57$) \dagger$ \\
\hline Model 2: HR $(95 \% \mathrm{Cl})$ & - & - & $1.09(1.07$ to 1.11$) \dagger$ & $1.54(1.52$ to 1.56$) \dagger$ \\
\hline
\end{tabular}

Model 1: adjusted for age and gender.

Model 2: adjusted for age, gender, hypertension, diabetes mellitus, previous stroke/TIA, vascular diseases, ESRD, COPD, autoimmune diseases, liver cirrhosis, dyslipidaemia, anaemia, CABG, AF, Charlson Comorbidity Index, ACEIs, ARBs, amiodarone, digoxin, beta-blockers and MRA.

*Number of events presented per 100 person-years of follow-up.

tCompared with high-income group.

ACEIs, ACE inhibitors; AF, atrial fibrillation; ARBs, angiotensin receptor blockers; CABG, coronary artery bypass graft; COPD, chronic obstructive pulmonary disease; ESRD, endstage renal disease; HF, heart failure; MRA, mineralocorticoid receptor antagonist (eplerenone/spironolactone); TIA, transient ischaemic attack.

age, lower rates of valvular heart or coronary artery bypass graft surgery in both low-income and median-income strata compared with the high-income HF group may reflect the fact that lowerincome patients with HF may remain poorly recognised or tend to seek medical help only when sicker. Furthermore, lowerincome HF populations were more likely to stay in suburban

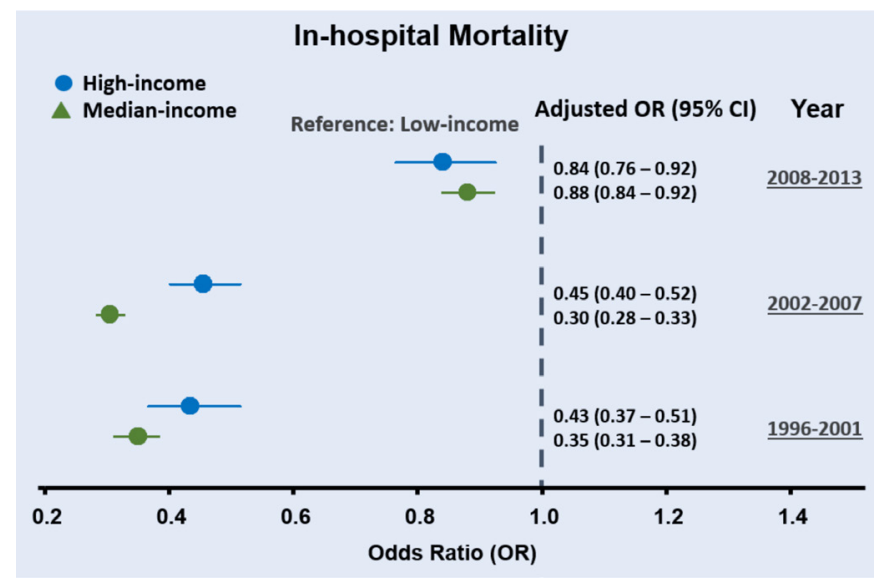

Figure 3 Temporal trend of in-hospital mortality stratified by three income groups. For in-hospital mortality, differences in in-hospital mortality for median-income and high-income groups compared with low-income heart failure group decreased over time (classified as 19962001, 2002-2007, 2008-2013). or rural areas, supporting effects of geographical variations and aggregated poverty, resulting in disparities in healthcare utilisation. ${ }^{19} 20$ Nevertheless, the observed differences in postdischarge HF outcome from socioeconomic disparities appeared to diminish about one decade following NHI programme implementation (figure 5).

Notably, we noticed that patients with HF with lower income showed a lower prescription rate of evidence-based GDMT for heart failure with reduced ejection fraction (HFrEF), ${ }^{21}$ including ACEIs/ARBs, BBs and MRA. ${ }^{22}$ Instead, prescription rate of digoxin or diuretics was substantially higher in low-income patients with HF despite their older age, higher clinical disease complexity and yet less prominent variations of prevalent atrial fibrillation compared with higher-status groups (all <20\%). ${ }^{23}$ This finding likely supported the gap in evidence-based HF practice and likely represents variations in prescription habits of healthcare providers, along with lower awareness on GDMT adherence in low-income patients with HF, especially in certain areas among Asian societies. Interestingly, income level has been proposed as an essential component of socioeconomic status influencing medication adherence in HF polypharmacy. ${ }^{24}$ Based on a more recent study, even suboptimal adherence to GDMT (ie, nearly half of the guideline-recommended dosage) has been shown to substantially improve HF outcomes. ${ }^{25}$ To the best of our knowledge, this study is the first to delineate the demographics of postdischarge HF survivors in a large-scale, population-based study examining the temporal associations of income level and GDMT use with postdischarge HF outcome following the 
A

HF Readmission

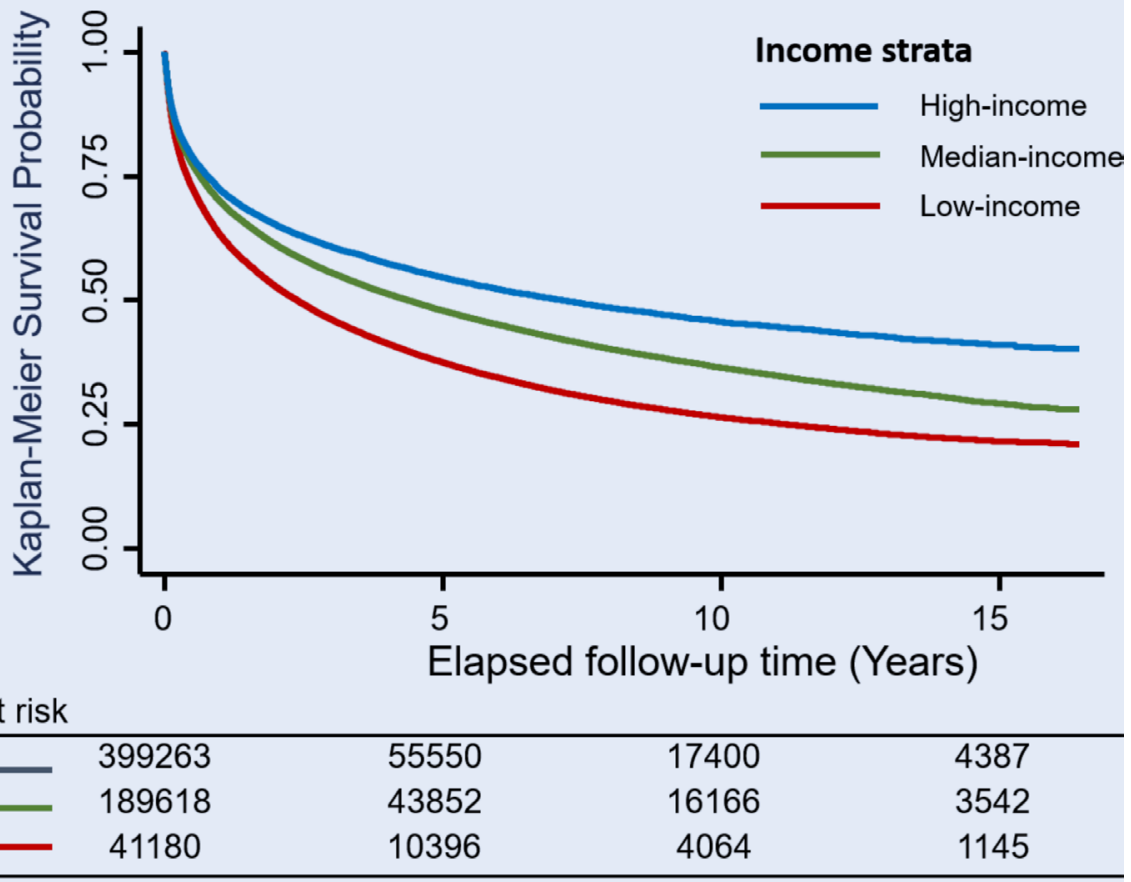

B

HF Readmission/All-cause Mortality

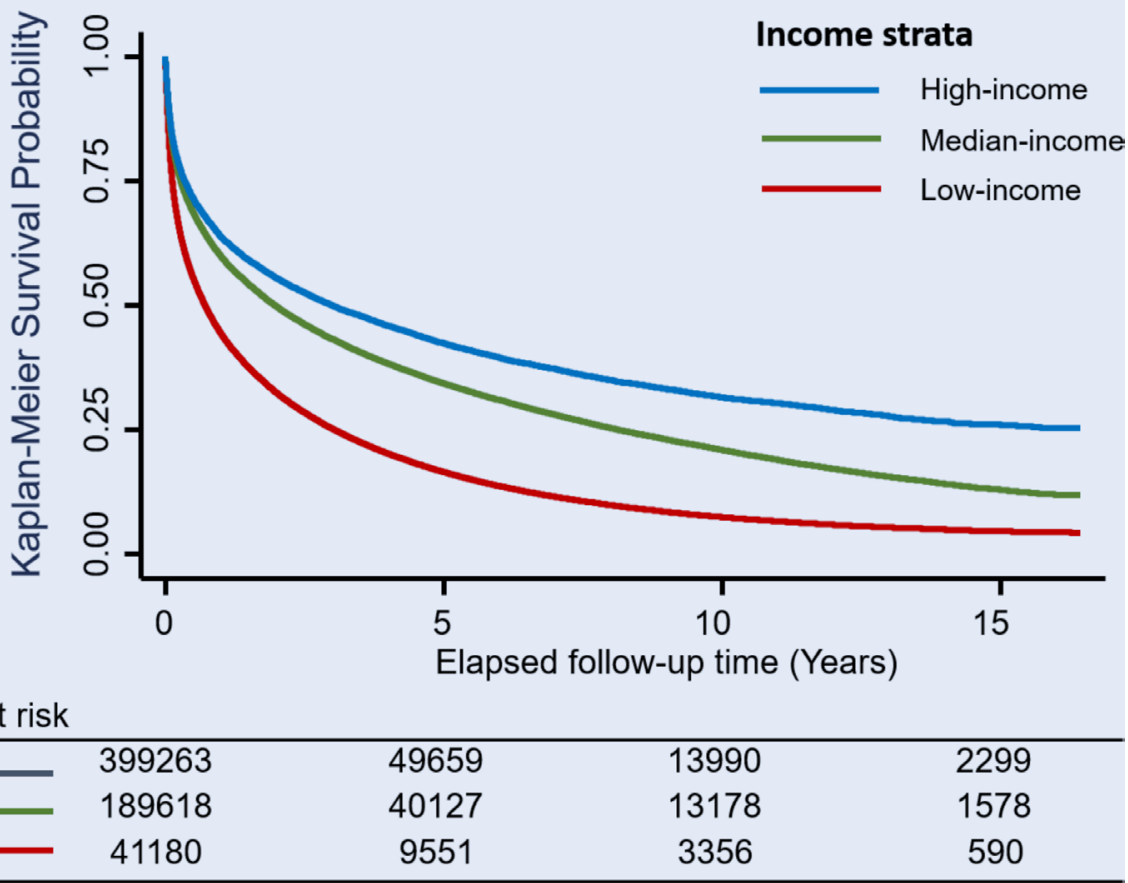

Figure 4 Heart failure (HF) readmission (A) and composite HF readmission/all-cause mortality (B) using Kaplan-Meier survival-free curves stratified by three income groups. Patients with HF of low income consistently demonstrated higher risk for postdischarge HF readmission or composite HF readmission/all-cause mortality compared with patients of higher-income strata. 


\section{Temporal Trends of HF Readmission and All-cause Mortality Incidence} by Income Categories over Years (1996 - 2013)

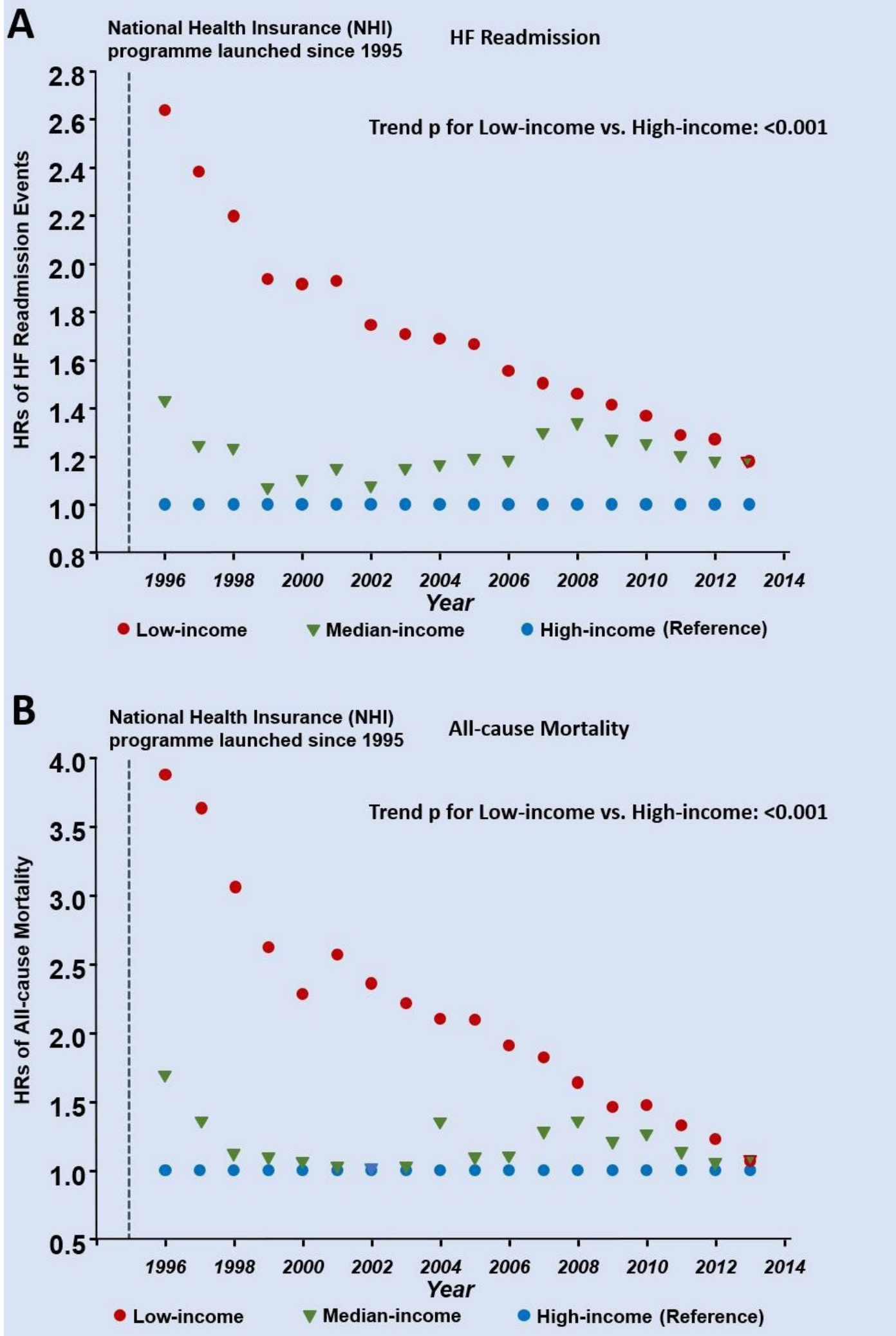

Figure 5 Temporal trends of heart failure (HF) readmission (A) and all-cause mortality (B) by three income groups over time (1996-2013). A marked decrease in the incidence of HF readmission and all-cause mortality was observed over time for the low-income group (expressed as HR, reference: high-income group). A linear trend analysis was used for adjusted HR for low-income versus high-income HF group (as reference) across observation time (per year as ordinal category). 
implementation of the nationwide universal healthcare coverage. The strength of the current study included data extraction from a healthcare system, providing $>99 \%$ coverage for all citizens less likely to be biased according to geographical variations, subpopulations/strata or degree of urbanisation tightly bound to income status, therefore disclosing real-world HF key features reflecting demographics, managements and outcomes by income strata with a relatively long span of follow-up time at the country level.

Patients of lower socioeconomic status may have lower chance of receiving evidence-based treatments due to financial pressures in a society without global healthcare coverage. ${ }^{26}$ As such, reform of the nationwide health insurance policies and integrations of multidisciplinary teams working (such as PostAcute Care (PAC) programme) with optimal discharge planning and referral system ${ }^{27-29}$ may theoretically improve the adherence of evidence-based HF therapy (ie, GDMT) based on the public health standpoint. Taken together, our findings highlight the potential benefits of implementing nationwide health insurance to overcome barriers to effective therapeutic interventions and thus to improve HF outcomes.

\section{Study limitations}

The analysis and findings of the current study were not without limitations. The data extracted from Taiwan's NHIRD did not contain information on the distinct HF phenotypes (reduced (HFrEF) or preserved ejection fraction HF); nevertheless, accumulating data have suggested that the rate of acute HF may distribute evenly in distinct phenotypes of HF with similar outcomes. ${ }^{30}$ Notably, although we controlled for several key baseline demographics, comorbidities and GDMT use, the impact of socioeconomic disparities on outcomes remained prominent across different income strata, implying potentially

\section{Key messages}

\section{What is already known on this subject?}

- Income level and socioeconomic status have shown to be prognostic factors in cardiovascular diseases, including heart failure (HF).

- Epidemiological transitions of guideline-directed medical therapy (GDMT) utilisation and postdischarge outcomes in patients with HF following implementation of universal health coverage remain largely unexplored.

\section{What might this study add?}

- Based on a nationwide data set, postdischarge patients with $\mathrm{HF}$ with lower income were less likely to receive GDMT, had a higher clinical comorbidity burden and significantly higher events when compared with median-income and high-income groups.

- Such differences among various income groups of patients with HF appeared to mitigate over time about one decade following initiation of the nationwide universal health coverage policy.

\section{How might this impact on clinical practice?}

- Our findings likely demonstrated the efficacy of implementing nationwide universal health coverage in HF management by eliminating the gap between barriers to guideline-directed medical resources, access to standardised treatment and improved healthcare quality over time. unmeasurable societal and patient-level confounders (eg, cultural backgrounds, health maintenance behaviour or lifestyle factors). Moreover, although we speculated that the observed reduction of the gap in HF outcomes may likely be attributable to the implementation of NHI programme, we could not preclude an influence for an overall improved systemic public health service and diminished gap in economic and social inequities that ultimately affect patients' prognosis. Furthermore, major advances in new pharmacological or interventional HF therapies (such as implantable cardioverter-defibrillator or cardiac resynchronisation therapy) may have resulted in overall enhanced quality of care.

\section{CONCLUSIONS}

Lower-income level is associated with lower utilisation of evidence-based pharmacological HF treatments with higher in-hospital death rates and poorer postdischarge outcomes. The observed worse postdischarge outcomes in lower-income patients with HF appeared to mitigate over time following the implementation of the nationwide universal health coverage. However, some caution should be exercised in interpreting these findings due to overall a variety of unmeasurable factors over time. Despite these, understanding these data as a temporal trend may probably provide future directions to improve healthcare policies and financing models regarding public health infrastructure, thereby aiming for better resource reallocation for healthcare policy makers.

\section{Author affiliations}

${ }^{1}$ Department of Medicine, Mackay Medical College, New Taipei City, Taiwan ${ }^{2}$ Division of Cardiology, Department of Internal Medicine, MacKay Memorial Hospital, Taipei, Taiwan

${ }^{3}$ Institute of Biomedical Sciences, Mackay Medical College, New Taipei City, Taiwan ${ }^{4}$ Division of Cardiology, Department of Medicine, Taipei Veterans General Hospital, Taipei, Taiwan

Institute of Clinical Medicine, and Cardiovascular Research Center, National YangMing University, Taipei, Taiwan

${ }^{6}$ General Clinical Research Center, Taipei Veterans General Hospital, Taipei, Taiwan ${ }^{7}$ Department of Medical Research and Education, Taipei Veterans General Hospital, Taipei, Taiwan

\section{Twitter Chung-Lieh Hung @CLHung}

Contributors C-LH, T-FC: coordination and helped draft the manuscript. C-HS, $\mathrm{H}-\mathrm{IY}$ : conceptual framework and reviewed the manuscript. J-NL, K-TS: performed the statistical analysis. C-EC: conceived of the study and participated in its design.

Funding The authors have not declared a specific grant for this research from any funding agency in the public, commercial or not-for-profit sectors.

\section{Competing interests None declared.}

\section{Patient consent for publication Not required.}

Ethics approval The present study was approved by the Institutional Review Board at Taipei Veterans General Hospital (2019-04-004AC), Taipei, Taiwan. Informed consent was waived due to anonymous data.

Provenance and peer review Not commissioned; internally peer reviewed.

Data availability statement This study is based in part on data from the National Health Insurance Research Database provided by the Bureau of National Health Insurance, Department of Health and managed by National Health Research Institutes. The interpretation and conclusions contained herein do not represent those of Bureau of National Health Insurance, Department of Health or National Health Research Institutes. Also, the data could not be spread or distributed out of the institution.

Supplemental material This content has been supplied by the author(s). It has not been vetted by BMJ Publishing Group Limited (BMJ) and may not have been peer-reviewed. Any opinions or recommendations discussed are solely those of the author(s) and are not endorsed by BMJ. BMJ disclaims all liability and responsibility arising from any reliance placed on the content. Where the content includes any translated material, BMJ does not warrant the accuracy and reliability of the translations (including but not limited to local 
regulations, clinical guidelines, terminology, drug names and drug dosages), and is not responsible for any error and/or omissions arising from translation and adaptation or otherwise.

Open access This is an open access article distributed in accordance with the Creative Commons Attribution Non Commercial (CC BY-NC 4.0) license, which permits others to distribute, remix, adapt, build upon this work non-commercially, and license their derivative works on different terms, provided the original work is properly cited, appropriate credit is given, any changes made indicated, and the use is non-commercial. See: http://creativecommons.org/licenses/by-nc/4.0/.

\section{ORCID iD}

Chung-Lieh Hung http://orcid.org/0000-0002-2858-3493

\section{REFERENCES}

1 Savarese G, Lund LH. Global public health burden of heart failure. Card Fail Rev 2017:3:7-11.

2 Conrad N, Judge A, Tran J, et al. Temporal trends and patterns in heart failure incidence: a population-based study of 4 million individuals. Lancet 2018;391:572-80.

3 Rajadurai J, Tse H-F, Wang C-H, et al. Understanding the epidemiology of heart failure to improve management practices: an Asia-Pacific perspective. J Card Fail 2017:23:327-39.

4 Ceia F, Fonseca C, Mota T, et al. Prevalence of chronic heart failure in southwestern Europe: the EPICA study. Eur I Heart Fail 2002;4:531-9.

5 Franks P, Winters PC, Tancredi DJ, et al. Do changes in traditional coronary heart disease risk factors over time explain the association between socio-economic status and coronary heart disease? BMC Cardiovasc Disord 2011;11:28.

6 Mensah GA. Heart failure in low-income and middle-income countries: rising burden, diverse etiology, and high mortality. J Card Fail 2018;24:833-4.

7 Obama B. United States health care reform: progress to date and next steps. JAMA 2016:316:525-32.

8 Lin L-Y, Warren-Gash C, Smeeth L, et al. Data resource profile: the National health insurance research database (NHIRD). Epidemiol Health 2018;40:e2018062.

9 Hsiao WC. Taiwan's path to universal health coverage-an essay by William C Hsiao. BMJ 2019;367:15979.

10 Hsieh C-Y, Su C-C, Shao S-C, et al. Taiwan's National health insurance research database: past and future. Clin Epidemiol 2019;11:349-58.

11 Lin C-C, Lai M-S, Syu C-Y, et al. Accuracy of diabetes diagnosis in health insurance claims data in Taiwan. J Formos Med Assoc 2005;104:157-63.

12 Charlson ME, Pompei P, Ales KL, et al. A new method of classifying prognostic comorbidity in longitudinal studies: development and validation. J Chronic Dis 1987:40:373-83.

13 Hawkins NM, Scholes S, Bajekal M, et al. Community care in England: reducing socioeconomic inequalities in heart failure. Circulation 2012;126:1050-7.
14 Wang SY, Tan ASL, Claggett B, et al. Longitudinal associations between income changes and incident cardiovascular disease: the Atherosclerosis risk in Communities study. JAMA Cardiol 2019;4:1203-12.

15 Dewan P, Rørth R, Jhund PS, et al. Income Inequality and Outcomes in Heart Failure: A Global Between-Country Analysis. JACC Heart Fail 2019;7:336-46.

16 Dokainish $\mathrm{H}$, Teo K, Zhu J, et al. Global mortality variations in patients with heart failure: results from the International congestive heart failure (INTER-CHF) prospective cohort study. Lancet Glob Health 2017;5:e665-72.

17 Callender T, Woodward M, Roth G, et al. Heart failure care in low- and middle-income countries: a systematic review and meta-analysis. PLoS Med 2014;11:e1001699.

18 Downing NS, Wang C, Gupta A, et al. Association of racial and socioeconomic disparities with outcomes among patients hospitalized with acute myocardial infarction, heart failure, and pneumonia: an analysis of within- and between-hospital variation. JAMA Netw Open 2018;1:e182044.

19 Soria-Saucedo R, Xu P, Newsom J, et al. The role of geography in the assessment of quality: evidence from the Medicare advantage program. PLoS One 2016;11:e0145656.

20 Auchincloss AH, Hadden W. The health effects of rural-urban residence and concentrated poverty. J Rural Health 2002;18:319-36.

21 Fonarow GC, Albert NM, Curtis AB, et al. Associations between outpatient heart failure process-of-care measures and mortality. Circulation 2011;123:1601-10.

22 Ponikowski P, Voors AA, Anker SD, et al. 2016 ESC Guidelines for the diagnosis and treatment of acute and chronic heart failure: The Task Force for the diagnosis and treatment of acute and chronic heart failure of the European Society of Cardiology (ESC)Developed with the special contribution of the Heart Failure Association (HFA) of the ESC. Eur Heart J 2016;37:2129-200.

23 Masoudi FA, Baillie CA, Wang Y, et al. The complexity and cost of drug regimens of older patients hospitalized with heart failure in the United States, 1998-2001. Arch Intern Med 2005;165:2069-76.

24 Allen LA, Fonarow GC, Liang L, et al. Medication initiation burden required to comply with heart failure guideline recommendations and hospital quality measures. Circulation 2015;132:1347-53.

25 Ouwerkerk W, Voors AA, Anker SD, et al. Determinants and clinical outcome of uptitration of ACE-inhibitors and beta-blockers in patients with heart failure: a prospective European study. Eur Heart J 2017:38:1883-90.

26 The HJ. Recession and health insurance coverage. Health Aff 2007-09;2011:145-52.

27 Feltner $C$, Jones $C D$, Cené CW, et al. Transitional care interventions to prevent readmissions for persons with heart failure: a systematic review and meta-analysis. Ann Intern Med 2014:160:774-84.

28 Albert NM, Barnason S, Deswal A, et al. Transitions of care in heart failure: a scientific statement from the American heart association. Circ Heart Fail 2015;8:384-409.

29 Riley JP, Masters J. Practical multidisciplinary approaches to heart failure management for improved patient outcome. Eur Heart J Suppl 2016;18:G43-52.

30 Senni M, Gavazzi A, Oliva F, et al. In-Hospital and 1-year outcomes of acute heart failure patients according to presentation (de novo vs. worsening) and ejection fraction. results from IN-HF outcome registry. Int J Cardiol 2014;173:163-9. 